\title{
Flare Irradiance Spectral Model (FISM): Flare component algorithms and results
}

\author{
Phillip C. Chamberlin, ${ }^{1}$ Thomas N. Woods, ${ }^{1}$ and Francis G. Eparvier ${ }^{1}$
}

Received 25 October 2007; revised 15 March 2008; accepted 22 March 2008; published 2 May 2008.

[1] The Flare Irradiance Spectral Model (FISM) is an empirical model developed for space weather applications that estimates the solar irradiance at wavelengths from 0.1 to $190 \mathrm{~nm}$ at $1 \mathrm{~nm}$ resolution with a time cadence of $60 \mathrm{~s}$. This is a high enough temporal resolution to model variations due to solar flares, where few accurate measurements at these wavelengths exist, as well as the solar cycle and solar rotation variations. The FISM modeling of the daily component variations, including variations from the solar cycle and solar rotation, was the topic of the first FISM paper (Chamberlin et al., 2007). The modeling of the FISM flare component that includes the solar irradiance variations from both the impulsive and gradual phases of solar flares is the topic of this paper. The flare component algorithms and results are discussed, and comparisons show that FISM estimates agree within the stated uncertainties with measurements of the solar vacuum ultraviolet (VUV; 0.1-200 $\mathrm{nm}$ ) irradiance. Results from FISM show that the relative change of the solar irradiance during flares for some wavelengths can exceed those of the solar cycle relative changes, ranging from factors of 60 times the quiet Sun irradiance during the gradual phase for emissions originating in the solar corona to factors of 10 in the transition region emissions during the flare's impulsive phase. FISM fully quantifies, on all timescales, the changes in the solar VUV irradiance directly affecting satellite drag, radio communications, as well as the accuracy in the Global Positioning System (GPS).

Citation: Chamberlin, P. C., T. N. Woods, and F. G. Eparvier (2008), Flare Irradiance Spectral Model (FISM): Flare component algorithms and results, Space Weather, 6, S05001, doi:10.1029/2007SW000372.

\section{Introduction}

[2] Space weather deals with how changes in the Sun, on timescales of minutes to decades, interact and drive changes in the heliophysics system. These variations cause significant deviations in the Earth and space environment on similar timescales, such as changes in the atmospheric densities and composition of particular atoms, molecules, and ions of Earth and other planets. The increase in the solar vacuum ultraviolet (VUV; 0.1-200 nm) irradiance deposits its energy into the thermosphere and mesosphere. Therefore, when there is a sudden increase in this irradiance due to a solar flare, there is increased heating in the upper atmosphere that causes this Earth's atmosphere to expand. This atmospheric expansion then leads to increased density at a given altitude, increasing the drag on satellites that are in low Earth orbit [Chamberlin et al., 2007]. The high-energy photons from the solar VUV irradiance also drive ionization of the neutral constituents

\footnotetext{
${ }^{1}$ Laboratory for Atmospheric and Space Physics, University of Colorado, Boulder, Colorado, USA.
}

in the upper atmosphere, creating the ionosphere. The ionization rate then increases when there is an irradiance increase from a solar flare, causing an increase in the density of electrons. Higher electron density can affect radio communications, and for large flare can even lead to radio communication blackouts. Increases in electron densities also degrade the accuracy in the highly relied upon Global Positioning System (GPS).

[3] Measurements of solar VUV irradiance represent a valuable data set that contributes to many aspects of research spanning the disciplines of space weather and the heliophysics system. Because there is a need for space-based instruments to observe wavelengths shortward of $320 \mathrm{~nm}$ due to atmospheric absorption, direct observations of the solar VUV irradiance were not possible until the first VUV spectrum from a sounding rocket was obtained in 1946 [Baum et al., 1946]. Since that first sounding rocket, there have been various instruments, both rocket and satellite based, that have sporadically observed different parts of the X-ray ultravi- 
olet (XUV; 0.1-10 nm), extreme ultraviolet (EUV; 10$120 \mathrm{~nm}$ ), and far ultraviolet (FUV; 121-200 nm) regions.

[4] The Solar EUV Experiment (SEE) [Woods et al., 1998a, 2005a] on the Thermosphere Ionosphere Mesosphere Energetics and Dynamics (TIMED) satellite is the first instrument to make subdaily solar irradiance measurements over the full EUV range since the Atmospheric Explorer-E (AE-E) satellite measurements in the late 1970s [Hinteregger et al., 1981]. There have also been various rocket flights to measure the solar EUV spectrum [Woods et al., 1998b], and also limited measurements of the solar EUV irradiance from the Solar and Heliospheric Observatory (SOHO) satellite. The SOHO Solar Extreme ultraviolet Monitor (SEM) instrument measures the 26-34 nm and the 0-50 nm irradiances with a time cadence of $1 \mathrm{~min}$ [Judge et al., 1998], and the SOHO Coronal Diagnostic Spectrometer (CDS) instrument measures the solar EUV irradiance with a time cadence of once per month [Thompson and Brekke, 2000]. While these instruments have provided some solar EUV measurements over the past 50 years, along with others like the Orbiting Solar Observatory (OSO) satellites in the 1960s and 1970s, there are still large data gaps in observations, and large uncertainties in some of these existing data. The EUV measurements are scheduled to continue with the Extreme Ultraviolet Variability Experiment (EVE) on the Solar Dynamics Observatory (SDO), with its launch planned for December 2008.

[5] The FUV irradiances have been monitored more frequently in recent history. These observations are mainly from two versions of the Solar Stellar Irradiance Comparison Experiment (SOLSTICE) that have flown on the Upper Atmospheric Research Satellite (UARS) from 1991 until 2005 and also on the Solar Radiation and Climate Experiment (SORCE) that launched in 2003 and continues to provide this measurement [Rottman et al., 1993]. The Solar Ultraviolet spectral irradiance monitor (SUSIM) on board UARS also provided measurements of the solar UV irradiance from 115 to $410 \mathrm{~nm}$ [Brueckner et al., 1993]. There are also measurements from 2002 up to the present of the FUV from TIMED SEE. The SEE FUV measurements, due to the accurate preflight and in-flight calibration techniques, have accuracies ranging from 2 to $10 \%$ [Woods et al., 2005a]. There are also more historical measurements of the FUV, mainly from the Atmospheric Explorer (AE-C, AE-D, and AE-E) satellites in the 1970s and the Solar Mesosphere Explorer (SME) from 1981 to 1989 .

[6] The XUV also contains many data gaps, as there is also a need for space-based measurements of these wavelengths due to the atmospheric absorption eliminating the ability for simplified ground-based observations. The current and recent historical measurements of the XUV have been obtained primarily on three satellites. The first recent XUV measurements were done with the Solar X-ray Photometer (SXP) on the Student Nitric Oxide
Explorer (SNOE) from 1997 until 2003 [Bailey et al., 1996, 2000]. Subsequent measurement of the XUV have been, and continue to be, made from two versions of the XUV Photometer System (XPS), one on the TIMED satellite [Woods et al., 1998a] and another on SORCE. The SOHO SEM also contains XUV measurements in its broadband central channel from 0.1 to $50 \mathrm{~nm}$, but this channel is dominated by wavelengths from the EUV. Despite the few current and historical measurements of the solar VUV irradiance, several challenges remain in fully understanding the VUV irradiance from only having broadband measurements in the $0-30 \mathrm{~nm}$ range and many data gaps, both in wavelength coverage and in time coverage.

[7] Various VUV irradiance models have been used to help fill the gaps between these measurements, such as the HFG model [Hinteregger et al., 1981], the EUVAC model [Richards et al., 1994, 2006], the Solar Irradiance Platform v2_30_63 (formerly SOLAR2000) model [Tobiska et al., 2000; Tobiska, 2004], VUV2002 [Woods and Rottman, 2002], the NRLEUV model [Warren et al., 1998, 2001; Lean et al., 2003] and a model by Kretzschmar [Kretzschmar et al., 2006]. These models, except for SOLAR2000, are all daily average models, meaning they do not have the ability to estimate the increased VUV irradiance from solar flares. The larger flares can cause the solar irradiance to increase 1000 times in the $X$ rays $(0.1-10 \mathrm{~nm})$, by factors of $2-10$ in the extreme ultraviolet (EUV; 10$120 \mathrm{~nm})$, and by 1.1-10 times in the far ultraviolet (FUV; 122-200 nm) wavelengths, as well as about a $20 \%$ effect for Lyman- $\alpha(121.6 \mathrm{~nm})$ [Woods et al., 2004]. SOLAR2000 has now been updated to produce the historical irradiances at a $3-\mathrm{h}$ temporal resolution, while also providing a new product, SOLARFLARE, that can now-cast (7-min latency) the solar VUV irradiance with a 2-min cadence [Tobiska, 2007].

[8] The Flare Irradiance Spectral Model (FISM) was developed to improve upon both the absolute accuracies of the model estimations based on newly available data sets, as well as to increase the temporal resolution to include the variations due to solar flares. To model the daily component variations, FISM uses data from various instruments on three different satellites. The daily averaged EUV irradiance data come from TIMED SEE, the FUV irradiance measurements from SEE as well as from the UARS SOLSTICE, while the XUV and shortwavelengths EUV measurements (less than $27 \mathrm{~nm}$ ) are provided by both the SEE and SORCE versions of the XPS [Woods et al., 2005b; Woods and Rottman, 2005]. The results shown here are the FISM Version 1, Revision 0 (FISM_1_0) results which are based on the TIMED SEE Version 9 data, UARS SOLSTICE Version 18, and SORCE XPS Version 8 products, but FISM will be updated to use the recent basis data sets as they are released and the revision increased. Major changes to FISM irradiance estimations and algorithms will be cause for a new 
release of the FISM data and the version number to be increased.

[9] The FISM daily component estimates, with respect to other models, provides a more accurate determination of the solar XUV, EUV, and FUV irradiances (0.1$190 \mathrm{~nm}$ ), showing better agreement with the recent measurements because FISM is based on TIMED SEE measurements. The FISM algorithms, along with the data sets and proxies that are used, resolve most of the discrepancies between the previously mentioned models and measurements provided by SEE, including the large solar cycle offsets and the solar rotation temporal discrepancies. The improvements of the daily model estimations provided by FISM were discussed in a previous paper [Chamberlin et al., 2007; hereinafter referred to as FISM1].

[10] The most significant improvement provided by FISM is that it can model the solar irradiance with up to a 3-s temporal resolution, although the standard output is currently at $60 \mathrm{~s}$. The previously discussed daily models neglect the very rapid, large-magnitude changes in the XUV, EUV, and FUV irradiance from large solar flares that can have a significant impact on Earth and are important for a variety of space weather applications. Not including flares can underestimate the integrated daily averaged solar irradiance from 0.1 to $120.0 \mathrm{~nm}$ by more than $13 \%$ for a day containing a single $\mathrm{X}$-class flare. The higher temporal scale for FISM will allow atmospheric models to calculate the effects of the changing solar irradiance on Earth more accurately and over shorter time intervals. The flare components of FISM are covered in this FISM2 paper.

\section{Data Sets}

[11] For FISM to successfully model the VUV irradiance response to flares, measurements of these events are needed to provide the basis for the model. There have been many flare observations in the EUV and FUV before, but most of the previous measurements were made with spectrometers that could only measure a single wavelength at a time. The SEE EUV Grating Spectrograph (EGS) provides more optimal observations of flares for EUV and FUV wavelengths than the spectrometer observations. The SEE EGS, being a spectrograph, has the advantage of observing the whole range from 27 to $194 \mathrm{~nm}$ at the same time. Combining the SEE and SORCE XPS diode measurements with the EGS measurements extend the range of concurrent observations from a prior lower limit of $27 \mathrm{~nm}$ down to $0.1 \mathrm{~nm}$. Therefore, all of the wavelengths and how they relate to each other at the similar times during the various stages of the flare can be studied simultaneously. The two channels of TIMED SEE, the XPS and the EGS, along with the SORCE XPS, have made fortuitous observations of 39 large flares at certain points in the flare evolution providing FISM with the necessary measurements to derive relations to the flare proxies. Included in these
39 large flares are 26 flares that were observed during the gradual phase and 13 flares that were observed during the impulsive phase.

[12] The SEE instrument has an inherent temporal resolution of $10 \mathrm{~s}$, and the median of these 10-s integrations over the approximate 3-min observation time are then used to make the SEE Level 3A orbit-averaged data product that is available to the public. The SEE Level 1 data are not publicly available as the short integration periods lead to noisier data, and various data processing corrections are not made until the Level 2 products. To make the Level 1 data useable and have the most accurate 10-s resolution observations that are critical for observing the large impulsive phase variations, the various Level 2 data processing corrections need to be made to the Level 1 data. The SEE Level 1 data is divided by the SEE Level 2A data for the previous orbit where no flare was observed to determine the correction factor or ratio. This correction factor is applied to the SEE Level 1 data during the flare observation as the data processing correction factors do not change appreciably in the short time from observations during one orbit to the next. This then corrects the Level 1 data resulting in a valid 10-s temporal resolution SEE Level 1 data product. The SEE data processing algorithms and calibration results are given by Woods et al. [2005a].

\section{Flare Proxies}

[13] To empirically model the flare variations, a representative proxy is needed that is on a high enough temporal resolution to observe the flare evolution, as well as one that has a significant historical data set and plans for continued future measurements to drive the modeled results. The X-ray sensor (XRS) on the Geostationary Operational Environmental Satellites (GOES) currently provides two measurements of soft X-rays irradiances on 3-s, 1-min, 5-min, and daily averaged time cadences [Garcia, 1994]. The 3-s time resolution GOES data, which are not currently available on the National Oceanic and Atmospheric Administration (NOAA) Space Environment Center (SEC) Web site, were privately obtained for use in FISM. The GOES 3-s data are planned to be a standard data product released by SEC in the near future, as it has been shown in the development of FISM, among other studies, to more accurately represent the flare irradiance variations. With the redundancy of the GOES satellites and the location of the two operational satellites at two different locations in geostationary orbit, the GOES 0.1 $0.8 \mathrm{~nm}$ data are very reliable with few data gaps since they began in the 1970s and should be available for many years to come.

[14] The short wavelength channel on GOES provides a value of the 0.05 to $0.4 \mathrm{~nm}$ integrated irradiance, while the long wavelength channel spans the 0.1 to $0.8 \mathrm{~nm}$ wavelength range. The GOES XRS was specifically designed to monitor these highly variable soft $X$-ray wavelengths that increase by orders of magnitude during large flares, as it 
also has the sensitivity range to capture many small flares. Because GOES XRS values are currently the only near real-time data that are given on timescales short enough to represent various changes in irradiance due to flares, the GOES $0.1-0.8 \mathrm{~nm}$ fluxes are used as the short-term solar flare proxy for FISM.

[15] There is question as to whether the coronal soft X-rays measured by the GOES XRS can accurately represent the EUV and FUV irradiance changes during a solar flare, as many emissions in the FISM model wavelength range are formed in different regions of the solar atmosphere containing various parts of the flare structure. The soft X-ray fluxes have been shown to correlate with the main-phase EUV irradiance changes from a flare [Priest, 1981; Horan et al., 1983]. This relationship has been shown for both the time duration of the flare and also the simultaneous time of the peak emission, but these results may also be attributed to inaccuracies or limitations of the observations. It was also initially derived by Neupert [1968], and then reconfirmed by Dennis and Zarro [1993] and many others, that the positive time derivative of the soft X-ray irradiance is an accurate temporal representation of the hard X-ray, impulsive phase of the flare. This relationship is now referred to as the "Neupert effect" and is important for FISM as the impulsive hard X-rays variations have been shown to correlate with the impulsive phase of EUV measurements, both temporally and energetically [McClymont and Canfield, 1986; Neupert, 1989]. Dennis and Zarro [1993] have performed further analysis on the temporal relationship and do show some spread in the timing of the impulsive phase peaks from the Hard X-Ray Burst Spectrometer (HXRBS) on the Solar Max Missions (SMM). Stated within this paper is that $88 \%$ of the events studied had hard X-ray peaks within $\pm 20 \mathrm{~s}$ of the time derivative soft $X$ rays, which is acceptable for FISM due to its nominal $60 \mathrm{~s}$ temporal resolution. Because no other proxy measuring an impulsive phase component is measured on a high enough time cadence and with a $100 \%$ duty cycle, the positive time derivative of the XRS 0.1-0.8 nm channel is also a flare proxy for FISM. The use of more representative proxies for the impulsive phase will become available in the near future when the NOAA GOES-N EUVS instrument becomes operational.

[16] The GOES change above the daily value, representing the gradual phase, and the time derivative of this irradiance change, representing the impulsive phase, are shown in Figure 1 for a flare on 3 November 2003. Since the GOES $0.1-0.8 \mathrm{~nm}$ soft X-ray irradiance has the ability to portray both the impulsive and gradual phases for the short-term flare enhancements, the GOES XRS data are used as the proxy for flare enhancements in the VUV range for FISM. Near real-time data products from Solar and Heliospheric Observatory (SOHO) Solar EUV Monitor (SEM) [Judge et al., 1998], GOES EUV Spectrometer (EUVS), and LYRA [Hochedez
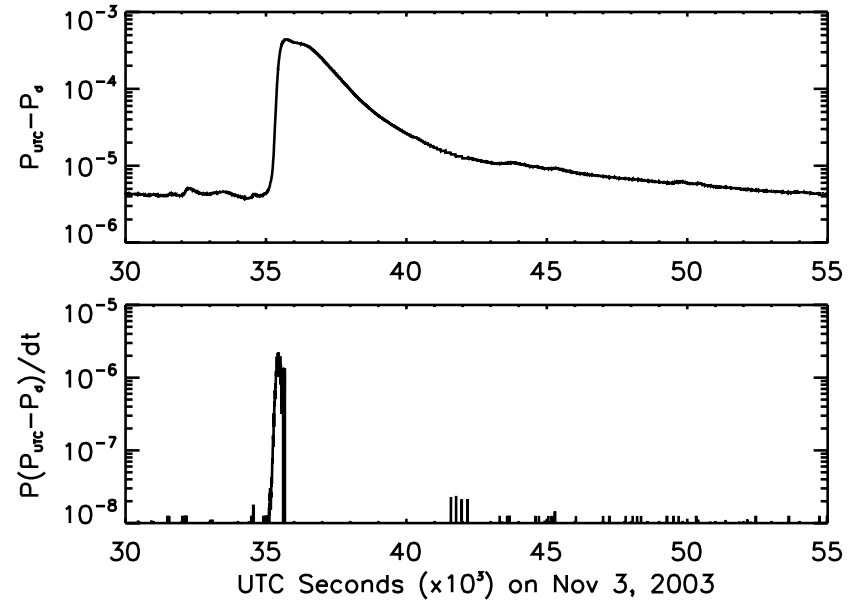

Figure 1. (a) The absolute change in irradiance of the GOES $1-8 \AA$ irradiance and (b) the time derivative of this change for a solar flare on 3 November 2003. These proxies temporally represent the gradual phase and the impulsive phases of flares for Flare Irradiance Spectral Model (FISM).

et al., 2006] in the future might prove useful as impulsive phase proxies with further study.

[17] It is known that significant center-to-limb variations (CLV) of active regions exist as they rotate around the observable solar disk [Worden et al., 2001]. Additionally, it has been found through analysis of SEE data that there are also CLV effects of solar flares in VUV wavelengths that need to be accounted for and modeled, as the CLVs are drastically different in flares for the soft X-ray proxy and for most of the VUV lines that are being modeled. This effect is seen in the comparison of the irradiance increase in the X17 flare on 28 October 2003 to the X28 flare on 4 November 2003, as shown in Figure 2. Both flares were fortuitously observed by SEE at times very near the observed GOES long channel irradiance peak. At the times of the SEE observation, the X28 flare is much larger in the soft $X$ rays, with a mean increase from 0 to $25 \mathrm{~nm}$ of $1240 \%$, than the X17 flare that has a mean increase of only $624 \%$ in the similar wavelength range. Contrary to the soft $X$ rays, the EUV flare variation is smaller for the X28 flare in the EUV than the increases observed by SEE during the X17 flare. The X28 flare occurred on the limb and the X17 flare was near disk center; consequently, center-to-limb darkening of the EUV emissions, center-to-limb brightening of the soft X-ray emissions, or contributions of both, can explain these differences in variations in the XUV and EUV ranges. The EUV irradiance increase over the daily median value is shown in Figure 2 for the X17 flare (black line) with an average increase of $91.8 \%$ for the 27-105 nm wavelength range. The X28 flare (gray line) occurred $7 \mathrm{~d}$ later (1/4 of a solar rotation) from the same active region responsible for the $\mathrm{X} 17$ flare; therefore, this 


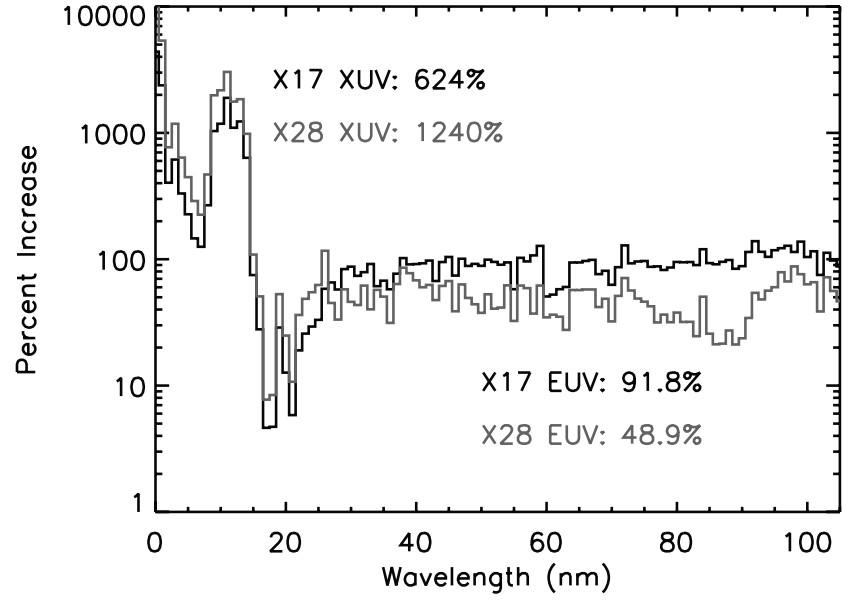

Figure 2. The percent increase in the $0-25 \mathrm{~nm}$ (XUV) and 27-105 nm (EUV) irradiance from the X17 flare occurring on 28 October 2003 and the X28+ flare on 4 November 2003. The EUV flare variation is smaller for the $\mathrm{X} 28+$ flare as compared to the EUV variation for the $\mathrm{X} 17$ flare, with the XUV wavelength increases reversed, due to the X17 flare being near disk center and the $\mathrm{X} 28+$ flare being on the limb.

flare occurred on the western limb of the Sun, and its EUV irradiance increase for the same EUV wavelength band is $48.9 \%$ as revealed in Figure 2. Because of the location of this flare on the solar limb, there is significant absorption of the enhanced EUV emission from the flare from the longer path through the solar atmosphere, and the EUV response is not as great. Therefore, the flare location on the solar disk is critical in determining how much of a correction due to the CLV needs to be applied, both in the initial stage to determine the accurate impulsive and gradual phase fit coefficients, as well as in the final stage to determine how the modeled irradiance for each wavelength is to be adjusted for CLV effects.

[18] The flare location input for FISM is determined from the "solar events reports" information available on the NOAA SEC Web page (www.sec.noaa.gov). The flare location information is derived using full disk solar images provided by the GOES Solar X-Ray Imager (SXI). Information is updated every $30 \mathrm{~min}$, so the CLV for flares has the potential to be applied at approximately the same time cadence. Even though a majority of the flares only form in the midlatitude regions of the Sun, both the longitude and the latitude are used in determining the most accurate heliocentric angle for the CLV correction in FISM.

\section{FISM Algorithms}

[19] The FISM concept to model the solar irradiance is that the irradiance for a particular wavelength $\lambda$ at time $t$, $\mathrm{E}(\lambda, \mathrm{t})$, has solar variations above a constant minimum irradiance value, $\mathrm{E}_{\min }(\lambda)$. Additional components added to the minimum irradiance value are the variations from the solar cycle, $\Delta \mathrm{E}_{\mathrm{SC}}$, solar rotation of active regions, $\Delta \mathrm{E}_{\mathrm{SR}}$ and also the impulsive and gradual phases of solar flares, $\Delta \mathrm{E}_{\mathrm{IP}}$ and $\Delta \mathrm{E}_{\mathrm{GP}}$. This relationship is given as:

$$
\begin{aligned}
\mathrm{E}(\lambda, \mathbf{t})= & \mathrm{E}_{\min }(\lambda)+\Delta \mathrm{E}_{\mathrm{SC}}(\lambda, \mathbf{t})+\Delta \mathrm{E}_{\mathrm{SR}}(\lambda, \mathbf{t}) \\
& +\Delta \mathrm{E}_{\mathrm{GP}}(\lambda, \mathbf{t})+\Delta \mathrm{E}_{\mathrm{IP}}(\lambda, \mathbf{t}) .
\end{aligned}
$$

The details, algorithms, and results in the modeling of the $\mathrm{E}_{\min }(\lambda), \Delta \mathrm{E}_{\mathrm{SC}}$, and $\Delta \mathrm{E}_{\mathrm{SR}}$ components that provide the FISM daily component estimations of the solar VUV irradiance were discussed in FISM1. The values for all coefficients that are stated in the FISM algorithms can be found on the FISM Web site (http://lasp.colorado.edu/ lisird/fism.htm) in order to reproduce the FISM model. The flare components, both the impulsive and gradual phase, are modeled as absolute values above the aforementioned daily component. Each type of irradiance variation is modeled separately, and the form and algorithms for each of the individual $\Delta \mathrm{E}$ flare components in equation (1) are discussed in the following sections.

\subsection{FISM Gradual Phase Flare Component}

[20] To construct an empirical model, a reference set of measurements is fit to the temporally related proxy values to initially determine the coefficients. As previously stated, the flare reference set of 39 flares was provided by the TIMED SEE and SORCE XPS measurements. The dates and SEE observation times of the flares are given in Table 1. Also given in this table are the GOES beginning, maximum, and end times, GOES class, and location of the flares on the solar disk determined by the GOES SXI.

[21] The GOES 0.1-0.8 $\mathrm{nm}$ irradiances at 3-s cadence are used in FISM as the proxy to represent the gradual phase of the solar flares. Because of the many orders of magnitude that the GOES values can change during a flare, flares are modeled as the absolute value of the irradiance minus the daily minimum irradiance. The daily "minimum" is actually taken to be the third lowest minimum irradiance value for the day to account for any anomalously low data points. This is necessary in the formulation for the flare component of FISM, rather than using relative ratios that the daily component uses. As an example, consider a GOES X1.0 flare $\left(1 \times 10^{-4} \mathrm{~W} / \mathrm{m}^{2}\right)$, and two cases when the daily minimum level is a GOES A1.0 $\left(1 \times 10^{-8} \mathrm{~W} / \mathrm{m}^{2}\right)$ or a $\mathrm{B} 1.0\left(1 \times 10^{-7} \mathrm{~W} / \mathrm{m}^{2}\right)$. The magnitude of the GOES irradiance change found by subtracting the daily minimum irradiance from the flare irradiance is essentially $10^{-4} \mathrm{~W} / \mathrm{m}^{2}$ for both examples and is still approximately the original value of the irradiance during large flares. On the other hand, if the flare algorithm used the relative change (ratio) over the daily minimum value, as in the FISM daily component algorithm, the ratio representation of the flare could fluctuate by an order of magnitude for flares of similar magnitude. 
Table 1. Large Flares Observed by TIMED SEE, Along With Various Parameters Derived From GOES XRS and SXI Data, During the Impulsive (Boldface) or Gradual Phase (Not Boldface) That Are Used As a Basis for Determining the FISM Flare Parameters

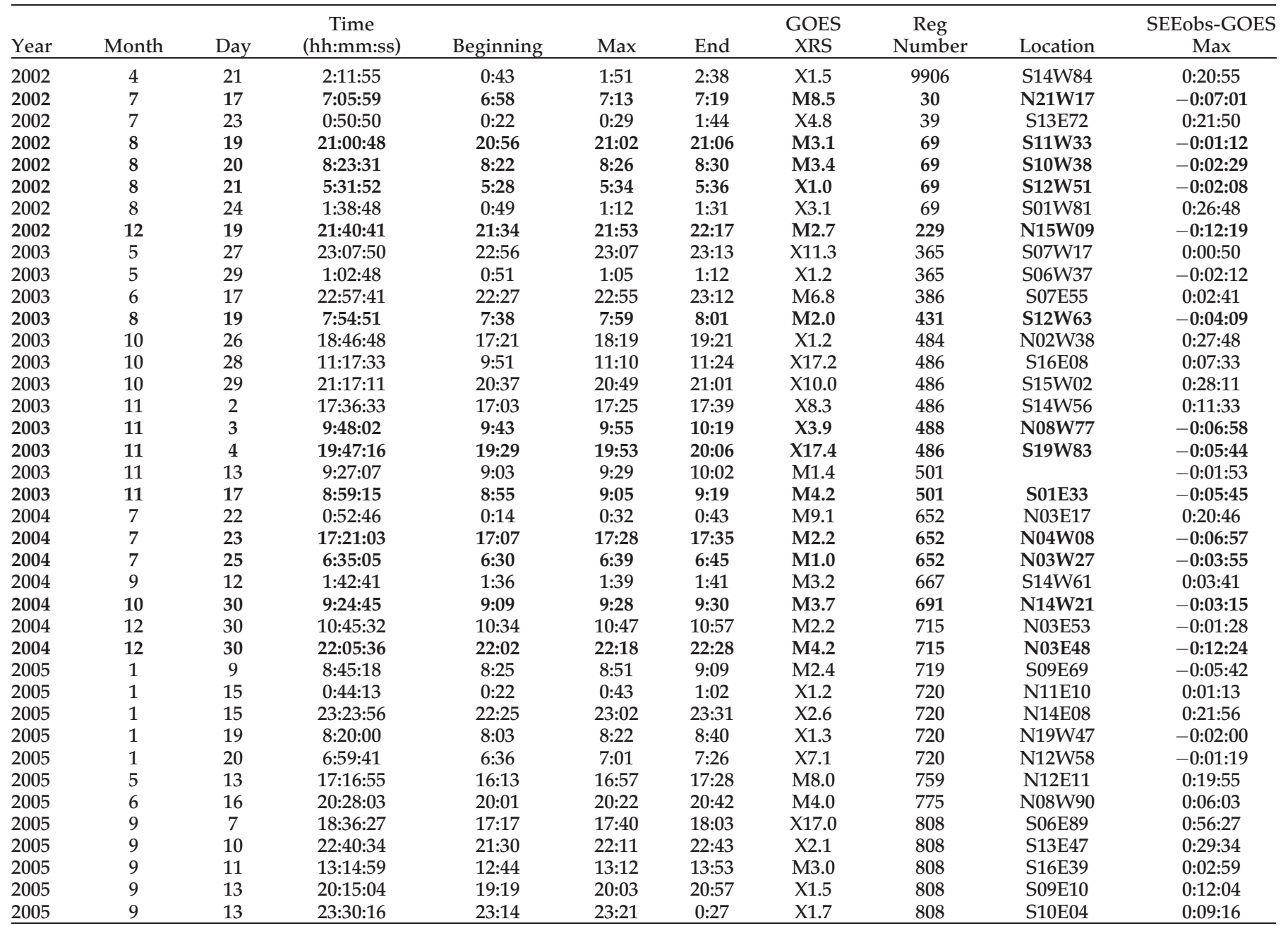

For the example, the flare ratio is 10,000 and 1000 for the same X1.0 flare. This large ratio variation results from dividing by a very small number in the denominator that can easily change by orders of magnitude on different days but does not have a significant contribution to the flare irradiance. This example clarifies why the flare proxy needs to be on an absolute scale instead of a relative ratio.

[22] As the flare component is the difference between the irradiance and its daily minimum value, for the measurements as well as the proxy, a power law relation was considered based on the large order of magnitude changes that occur in GOES XRS irradiances. It was determined that the best fit for the soft $X$ rays at wavelengths less than $14 \mathrm{~nm}$ is a linear fit, which is expected as both the measurement and the proxy are dominated by the same soft X-ray free-free emission. For the wave- lengths greater than $14 \mathrm{~nm}$, a power law provided a better fit to the data. The gradual phase flare algorithm is

$$
\begin{aligned}
\Delta \mathrm{E}_{\mathrm{GP}}\left(\lambda, \mathbf{t}_{\mathrm{UTC}}\right) & \equiv \mathrm{E}\left(\lambda, \mathbf{t}_{\mathrm{UTC}}\right)-\mathrm{E}_{\mathrm{d}}\left(\lambda, \mathbf{t}_{\mathrm{d}}\right) \\
& =\mathrm{C}_{\mathrm{GP}}(\lambda)\left[\mathrm{P}\left(\mathbf{t}_{\mathrm{UTC}}\right)-\mathbf{P}_{\mathbf{d}}\left(\mathbf{t}_{\mathbf{d}}\right)\right]^{\mathrm{N}_{\mathrm{GP}}(\lambda)} .
\end{aligned}
$$

$\mathrm{N}_{\mathrm{GP}}(\lambda)$ equals 1 for the soft $X$-rays $(\lambda<14 \mathrm{~nm})$, and therefore equation (2) is then just a linear equation, while the $\mathrm{N}_{\mathrm{GP}}(\lambda)$ average for the EUV wavelengths $(\lambda>14 \mathrm{~nm})$ is 0.647. $P_{d}\left(t_{d}\right)$ and $E_{d}\left(\lambda, t_{d}\right)$ are the daily minimum proxy and irradiance measurement for the day $t_{d} \cdot P\left(t_{\text {utc }}\right)$ and $E\left(\lambda, t_{\text {utc }}\right)$ are then the proxy and irradiance during a specific time, $t_{\mathrm{UTC}}$, in UTC seconds of the day.

[23] Equation (2) represents the relationship of the absolute change in the irradiance and proxy at a certain time from their respective daily minimum value. $E\left(\lambda, t_{u t c}\right)$ is actually an approximate $3 \mathrm{~min}$ average if using the SEE 
Level $3 \mathrm{~A}$ data or a $10 \mathrm{~s}$ measurement if using the SEE Level 1 data, centered at $t_{\text {UTC. }}$. The power law exponent of 0.647 for the EUV wavelength bins is an average that is derived by initially fitting the exponent, $\mathrm{N}_{\mathrm{GP}}(\lambda)$, as a variable along with the slope coefficient, $\mathrm{C}_{\mathrm{GP}}(\lambda)$. The value of the exponents from the initial fit are essentially constant for the EUV wavelengths, so the mean of the exponents from the two variable fits to the wavelengths from 30 to $110 \mathrm{~nm}$ is then set as the power for all wavelengths greater than $14 \mathrm{~nm}$. There were larger uncertainties in the FUV fits, where there are not significant changes from flares; therefore, setting the power exponent to a constant value helps constrain the fit for other wavelengths. The shortest wavelengths $(0-14 \mathrm{~nm})$ have a power of approximately 1 , proving that a linear fit is more ideal for these wavelengths, as expected.

[24] As discussed in section 3, there is a significant CLV effect for flares that needs to be accounted for in FISM. To determine the CLV function for each wavelength, there are actually three fits performed using equation (2), based on the location of the flare. The first fit is performed for flares that occurred with $\mu$ values between 1 and 0.76 , where $\mu=\cos (\theta)$, and $\theta$ is the flare's heliocentric angle determined from GOES SXI images. This first coefficient from the fit to the flares that are centrally located on the solar disk is labeled as $\mathrm{C}_{\mathrm{GP}, \mathrm{C}}(\lambda)$. The next fit to find $\mathrm{C}_{\mathrm{GP}, \mathrm{L}}(\lambda)$ is performed using equation (2) for flares located on or near the limb of the Sun, where $\mu$ is between 0.34 and 0 . The remaining flares are fit to those with $\mu$ values between 0.76 and 0.34 to determine $\mathrm{C}_{\mathrm{GP}, \mathrm{M}}(\lambda)$. The midlocation flare fit is primarily used to verify that $\mathrm{C}_{\mathrm{GP}, \mathrm{M}}(\lambda)$ does indeed fall between the central and limb flare fits, $\mathrm{C}_{\mathrm{GP}, \mathrm{C}}(\lambda)$ and $\mathrm{C}_{\mathrm{GP}, \mathrm{L}}(\lambda)$. Figure 3 is an example of the three power law fits that were found and the flares used in determining the fit for the $30.5 \mathrm{~nm}$ wavelength bin. It shows that the fit to the flares located in the center of the disk produces a much higher irradiance change in the EUV than the fit to the flares located on the limb of the solar disk.

[25] Once the fits are performed, and $\mathrm{C}_{\mathrm{GP}, \mathrm{L}}(\lambda)$ and $\mathrm{C}_{\mathrm{GP}, \mathrm{C}}(\lambda)$ are determined, then the coefficient for the gradual phase algorithm to be used for CLV corrected irradiance estimations, $\mathrm{C}_{\mathrm{GP}}(\lambda)$, is determined by fitting a normalized cosine function using the two values of $\mathrm{C}_{\mathrm{GP}, \mathrm{C}}(\lambda)$ and $\mathrm{C}_{\mathrm{GP}, \mathrm{L}}(\lambda)$, given by

$$
\mathrm{C}_{\mathrm{GP}}(\lambda)=\left[0.85 \mathrm{C}_{\mathrm{GP}, \mathrm{L}}(\lambda)\right]+\mu\left[1.06 \mathrm{C}_{\mathrm{GP}, \mathrm{C}}(\lambda)-0.85 \mathrm{C}_{\mathrm{GP}, \mathrm{L}}(\lambda)\right] .
$$

The $\mathrm{C}_{\mathrm{GP}}(\lambda)$ from equation (3) is the coefficient that is to be used in equation (2) that then scales the fit to account for the CLV based on the flare location given by $\mu$. As $\mathrm{C}_{\mathrm{GP}, \mathrm{L}}(\lambda)$ and $\mathrm{C}_{\mathrm{GP}, \mathrm{C}}(\lambda)$ were found using fits to sets of flares that were not exactly at $\mu=1$ and $\mu=0$, a correction factor must also be used to correct for the average $\mu$ values for the respective $\mu$ bins of 0.93 and 0.17 . The correction factor is 0.85 to correct the limb

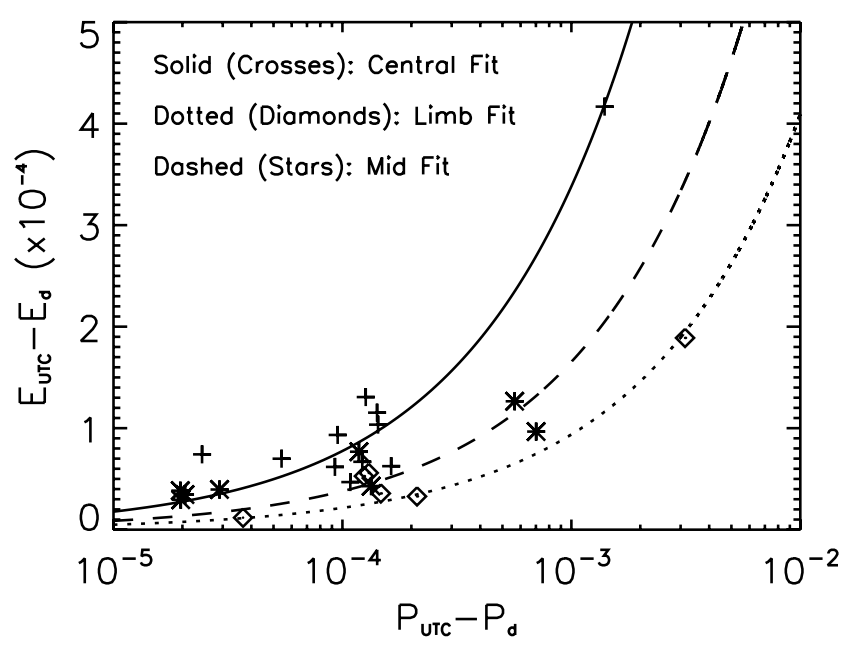

Figure 3. The power law fits for the $30.5 \mathrm{~nm}$ bin, with an exponent of 0.647 , for the gradual phase component. There are three fits performed to derive a CLV function. The fit to the centrally located flares (plus symbols), whose $\mu$ values range between 1.0 and 0.76 , is given by the solid line. The limb flares (diamond symbol) range from $\mu=0.34$ to $\mu=0.0$, and the fit is shown as the dotted line. The fit to the remaining flares, ranging from $\mu$ of 0.34 to $\mu$ of 0.76 (asterisk symbol), is shown as the dashed line.

coefficient to $\mu=0$ and 1.06 to correct the central coefficient to $\mu=1$.

[26] Equation (3) can also be manipulated algebraically into a different form given as

$$
\mathrm{f}(\mu, \lambda) \equiv \frac{\mathrm{C}_{\mathrm{GP}}(\lambda)}{1.06 \mathrm{C}_{\mathrm{GP}, \mathrm{C}}(\lambda)}=\operatorname{LCR}_{\mathrm{rat}}(\lambda)+\mu\left[1-\mathrm{LCR}_{\mathrm{rat}}(\lambda)\right]
$$

where the limb-to-center ratio, $\operatorname{LCR}_{\text {rat }}(\lambda)$, is the ratio of the coefficient from the flares near the limb $(\mu=0)$ to the coefficient from the flares located near disk center $(\mu=1)$, or

$$
\operatorname{LCR}_{\text {rat }}(\lambda)=\frac{0.85 \mathrm{C}_{\mathrm{GP}, \mathrm{L}}(\lambda)}{1.06 \mathrm{C}_{\mathrm{GP}, \mathrm{C}}(\lambda)} .
$$

A plot of $\operatorname{LCR}_{\text {rat }}(\lambda)$ is given in Figure 4 showing the strong center-to-limb darkening for solar flares in EUV wavelengths. Equations (4) and (5) provide a more meaningful CLV correction, and $\operatorname{LCR}_{\text {rat }}(\lambda)$ is then in a form that can be easily used in the impulsive phase CLV correction described later in this section.

[27] Once the CLV corrected gradual phase coefficient, $\mathrm{C}_{\mathrm{GP}}(\lambda)$, is determined, then the change in the irradiance from the flare during the gradual phase, $\Delta \mathrm{E}_{\mathrm{GP}}\left(\lambda, \mathrm{t}_{\mathrm{UTC}}\right)$, can be calculated whenever the proxy value is known at time $\mathrm{t}_{\mathrm{UTC}}$. The $\Delta \mathrm{E}_{\mathrm{GP}}\left(\lambda, \mathrm{t}_{\mathrm{UTC}}\right)$ is used in equation (1) to add 


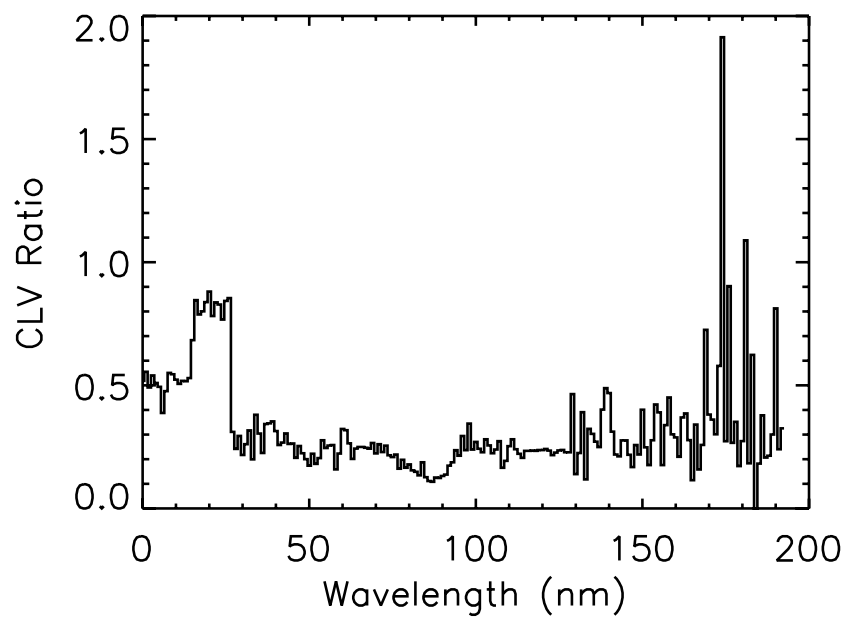

Figure 4. The ratio of the gradual phase coefficients from the flares located near the limb to the coefficients from the centrally located flares, or $L_{C R}$ rat. This CLV ratio shown here is convolved with the CLV of the GOES 0.1-0.8 nm data, so it does not represent the actual flare CLV for the VUV irradiances.

on the gradual phase flare component to the FISM modeled irradiance, $E(\lambda, t)$.

\subsection{FISM Impulsive Phase Flare Component}

[28] Given the relationship of the Neupert effect [Neupert, 1968] that states the time derivative of the gradual phase of the flare gives the temporal profile of the impulsive phase of the flare, the FISM impulsive phase algorithm is

$$
\begin{aligned}
\Delta \mathrm{E}_{\mathrm{IP}}\left(\lambda, \mathrm{t}_{\mathrm{UTC}}\right) \equiv & {\left[\mathrm{E}\left(\lambda, \mathbf{t}_{\mathrm{UTC}}\right)-\mathrm{E}_{\mathrm{d}}\left(\lambda, \mathrm{t}_{\mathrm{d}}\right)\right] } \\
= & \mathbf{f}(\mu, \lambda) \bullet \mathrm{C}_{\mathrm{IP}}(\lambda)\left[\frac { \mathrm { d } } { \mathrm { dt } } \left(\mathrm{P}\left(\mathbf{t}_{\mathrm{UTC}}\right)\right.\right. \\
& \left.\left.-\mathrm{P}_{\mathrm{d}}\left(\mathrm{t}_{\mathrm{d}}\right)\right)>5 \times 10^{-10}\right]^{\mathrm{N}_{\mathrm{IP}}(\lambda)}
\end{aligned}
$$

The irradiance and proxy values are once again given as the measured values at time $t_{\text {UTC }}$ and the daily minimum values for day $t_{d}$, as they were for equation (2). The CLV correction, $\mathrm{f}(\mu, \lambda)$, is derived from the gradual phase $\operatorname{CLV}_{\text {rat }}(\lambda)$, and is explained further in the following paragraphs. The derivative of the GOES proxy irradiance is limited to be greater than $5 \times 10^{-10}$ to have variations only during the rise of the gradual phase and to eliminate minor fluctuations of the derivative that are attributed to measurement noise and not actual solar variability.

[29] In flares there can be simultaneous contributions from the impulsive phase and the gradual phase of the flare, and hence at times the SEE data can include contributions from both phases of the flare. For the gradual phase contributions not to influence the derivation of the impulsive phase parameters in the model, the gradual phase component is first modeled, and then the modeled gradual phase contributions are subtracted from the SEE data. The residual thus represents only the impulsive phase of the flares. The FISM impulsive phase proxy has no gradual phase contributions because the GOES proxy representation is the time derivative of the GOES X-ray data.

[30] As there are only 13 large flares observed by SEE during the impulsive phase that are strong enough to be used in the modeling of FISM, including only two flares near the limb, the CLV function for the impulsive phase cannot be accurately determined in a similar way as the gradual phase CLV function. Therefore, the same CLV correction for the gradual phase is assumed for the impulsive phase. This CLV correction is applied to the SEE measurements to correct them to the center of the solar disk $(\mu=1)$ before the fit of equation (6) is performed to determine $\mathrm{C}_{\mathrm{IP}}(\lambda)$ and $\mathrm{N}_{\mathrm{IP}}(\lambda)$. The fit is done using $\mathrm{f}(\mu)$ given by equation (5). Figure 5 shows the impulsive phase fit to the CLV corrected EUV irradiance data at $139.5 \mathrm{~nm}$. Each color represents a different flare that was observed in the impulsive phase.

[31] As was done for the gradual phase power law fit, the exponent of the impulsive phase, $\mathrm{N}_{\mathrm{IP}}(\lambda)$ in equation (6), is also initially found but then set to be a constant value for all wavelengths. The constant value for the impulsive phase for EGS wavelengths (27-193) is the mean of the fit powers found from 60 to $110 \mathrm{~nm}$, and was determined to be 0.587 . All wavelengths shortward of $60 \mathrm{~nm}$ and longward of $110 \mathrm{~nm}$ were not used, as the central portion of the VUV spectrum corresponding to emissions from the transition region was observed to

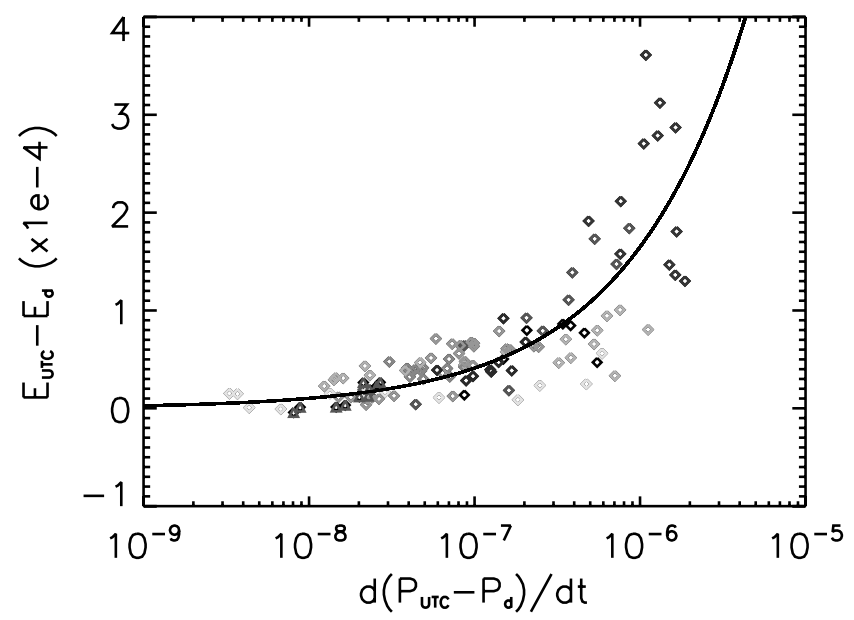

Figure 5. The impulsive phase component fit for the irradiance at $139.5 \mathrm{~nm}$ from 13 large flares. Each data point represents the SEE 10-s irradiance change at time $t_{U T C}$ from the daily minimum value as a function of the time derivative of the increase in the GOES 0.1-0.8 nm irradiance at $t_{\text {UTC }}$ from its daily minimum value. Each shade of gray represents a different flare. The black line represents the fit, which is a power law with 0.587 as the value of the exponent. 
show the largest response to the flares during the impulsive phase, and therefore should have a better fit. The fit of equation (6) is then repeated, this time using $\mathrm{N}_{\text {IP }}(\lambda)$ equal to 0.587 , and solving for the final value of $\mathrm{C}_{\mathrm{IP}}(\lambda)$.

[32] Once $\mathrm{C}_{\mathrm{IP}}(\lambda)$ has been determined, the impulsive phase flare component, $\Delta \mathrm{E}_{\mathrm{IP}}\left(\lambda, \mathrm{t}_{\mathrm{UTC}}\right)$, is estimated using equation (7), when proxy values are available. The GOES data are smoothed by a data point on each side to eliminate some of the noise in the GOES 3-s data before taking the time derivative for the impulsive phase proxy. The impulsive phase component is the last of the irradiance components in FISM that are added to obtain the complete modeled irradiance, $\mathrm{E}\left(\lambda, \mathrm{t}_{\mathrm{UTC}}\right)$, for a particular $1 \mathrm{~nm}$ wavelength bin, $\lambda$, at a particular time, $t_{\mathrm{UTC}}$.

[33] It has been noted previously in discussions about the GOES XRS data that there is little or no impulsive phase component in the soft $X$ rays. The lack of impulsive phase in the XUV can also be seen in Figure 6. This shows the SEE EGS Level 1 data (10-s integrations) for the integrated 154.75-155.15 nm C IV wavelength time series compared to the irradiance from the SEE XPS photometer measuring coronal wavelengths from 0.1 to $7 \mathrm{~nm}$. The bright C IV FUV emission shows the timing of the impulsive phase, while the XPS soft $X$ ray only show the initial rise of the gradual phase for these XUV wavelengths. The current Version 9 TIMED SEE XPS data processing algorithm uses the ratio of the GOES XRS channels to determine a temperature of the plasma, which then uses this temperature to drive the CHIANTI solar model to produce irradiance results from 0.1 to $40 \mathrm{~nm}$. The CHIANTI output is then scaled so that the integrated irradiance from 0.1 to $7 \mathrm{~nm}$ matches the irradiance from the 0.1-7 $\mathrm{nm}$ XPS diode, and then this scaled CHIANTI data is then used for the TIMED SEE Version 9 data from the entire wavelength range from 0.1 to $27 \mathrm{~nm}$ [Woods et al., 2008]. Even though there are impulsive phase flare contributions at wavelengths in the XPS region, for example the impulsive phase ribbons that have been seen in TRACE and EIT data at $19.5 \mathrm{~nm}$ and $17.1 \mathrm{~nm}$, this XPS data processing technique will eliminate any impulsive phase contributions that may be present at wavelengths from 7 to $27 \mathrm{~nm}$ as they are modeled output from 0.1 to $7 \mathrm{~nm}$ that has been shown in Figure 6 to not have any impulsive phase contributions. With the lack of impulsive phase emissions showing up in the TIMED SEE XPS version 9 data due to the data processing technique, there will be no impulsive phase emissions showing up in the FISM estimations from 0 to $27 \mathrm{~nm}$ either. This will be improved with the SDO EVE measurements.

[34] This observational evidence of the lack of existence of an impulsive phase increase in the 0.1-7 $\mathrm{nm}$ XPS channel 1 can be explained theoretically. The beam of accelerated particles triggered by the magnetic reconnection at the onset of a flare does not interact or produce enhanced emissions in the corona due to the low density

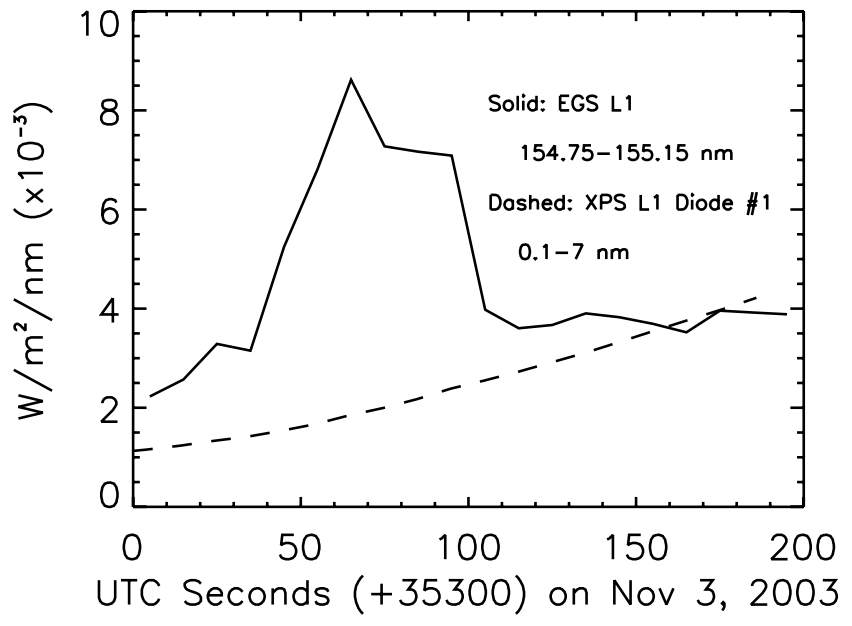

Figure 6. Time series of the transition region emission $(\mathrm{C} I V, \log (\mathrm{T})=5.00)$ from the EGS Level 1 data with wavelengths from 154.75 to $155.15 \mathrm{~nm}$ irradiance in the FUV (solid line) and the XPS photometers 1 broadband soft X-ray irradiance from 0.1 to $7 \mathrm{~nm}$ (dashed line) for a X 3.9 flare that had a GOES XRS peak at 35,700 s on this day (or $400 \mathrm{~s}$ on this plot). The large impulsive phase response can be seen in the C IV FUV transition region emission, but the coronal soft $X$ rays show no impulsive phase response, only the initial rise from the gradual phase of the flare.

of the corona. The newly accelerated particles travel freely down the flaring magnetic flux tube with very little Coulomb or collisional interactions with their surrounding plasma. It is not until the particle beam encounters the much higher-density transition region and chromosphere that there are significant interactions with the surrounding plasma to produce enhanced emissions during the impulsive phase. The gradual phase is then the heating of the entire solar atmosphere due to chromospheric evaporation of the higher-temperature plasma caused by the deposited energy from these accelerated particles.

\subsection{FISM Uncertainty}

[35] The uncertainty for the modeled FISM spectra is determined separately for the daily components and the flare components. The FISM daily component uncertainty is discussed in the FISM1 paper. The FISM flare component uncertainty is calculated by finding the weighted standard deviation for the impulsive phase and the gradual phase components separately using:

$$
\sigma(\lambda)=\sqrt{\frac{\sum_{\mathbf{i}=0}^{n}\left[\frac{E_{\text {Mod }}(i, \lambda)-E_{\text {Meas }}(i, \lambda)}{E_{\text {Meas }}(i, \lambda)}\right]^{2}}{n-1}} .
$$




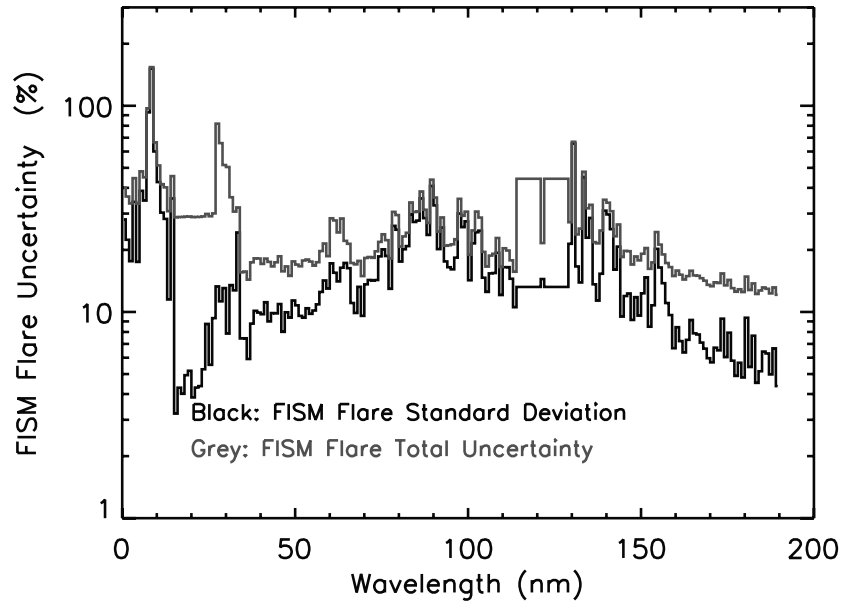

Figure 7. The FISM uncertainties for the combined daily and flare components (black) as well as the FISM total uncertainty that combines the FISM uncertainties with the SEE accuracy. The large uncertainties in the short XUV wavelengths are from the large variations that occur during the gradual phase. The increased uncertainties in the mid-VUV wavelengths $(70-100 \mathrm{~nm})$ are from the larger variations that occur at these wavelengths during the impulsive phase of the flare.

After both the impulsive and gradual phase component uncertainties are found, the standard deviations are added in quadrature, or

$$
\sigma_{\text {Flare }}=\sqrt{\sigma_{\mathrm{GP}}^{2}+\sigma_{\mathrm{IP}}^{2}} .
$$

The impulsive phase component uncertainties are found using the same SEE Level 1 corrected data that were used in determining the impulsive phase fit for $E_{\text {Meas }}$ and likewise the gradual phase component uncertainties are also found using the SEE data that were used to determine the gradual phase fit for $\mathrm{E}_{\text {Meas }}$ but in this case it is the SEE Level $3 \mathrm{~A}$ data. The difference in using the SEE Level 3A (3-min) for the gradual phase versus the Level 1 corrected data (10-s) is again from the large irradiance changes that occur over a shorter period during the impulsive phase. The uncertainty calculation used the FISM estimations and the SEE data from all flares given in Table 1 to determine the FISM flare uncertainty.

[36] The "uncertainty" refers to the ability of FISM to reproduce the base data set. For example, how well the FISM gradual phase flare component at $33.5 \mathrm{~nm}$ represents the SEE Level 3A value at the same wavelength. The FISM "total uncertainty" is the FISM uncertainties added in quadrature with the uncertainties of the base data set, namely the accuracy of the SEE measurements.

\section{FISM Flare Results}

[37] Even with the relatively small number of absolutely calibrated observations of flares spanning the VUV spec- trum, FISM uses the available observations to accurately model the VUV irradiance changes during a solar flare for both the impulsive and gradual phases. The FISM uncertainties for the flare components are shown in Figure 7, along with the FISM flare total uncertainty that incorporates the SEE L3A data product accuracy. There are large FISM uncertainties in the shortest XUV wavelengths, just above $100 \%$, but then the total uncertainty quickly decreases to below $40 \%$ for the rest of the VUV wavelengths greater than $14 \mathrm{~nm}$. The large uncertainties shortward of $4 \mathrm{~nm}$ are a result of the order of magnitude increases of these wavelengths during the gradual phase of a solar flare and also because of the small number of measurements available from SEE to base FISM on. There are also larger uncertainties in the irradiances between 60 and $160 \mathrm{~nm}$, as seen in Figure 7, from the uncertainty contributions from the impulsive phase of solar flares. While more flare data can help reduce the FISM flare uncertainties, the FISM flare results are a significant improvement over models with just daily components.

[38] The relative difference between the average FISM spectrum for the day (using the 60-s data containing the flare estimations) and the daily component irradiance is computed for each day from 1 January 2003 until 31 December 2005 and is shown in Figure 8 as the flare contribution for each day. The two wavelength bands used for this comparison are important bands that are often used by the space weather community in atmospheric simulations and models. The two bands are the XUV wavelengths and the integrated XUV and EUV wavelengths from 0 to $105 \mathrm{~nm}$. These plots show that the daily components underestimate the daily irradiance contributions by less than $5 \%$ for most days when there are no large flares. During a day with multiple large flares,
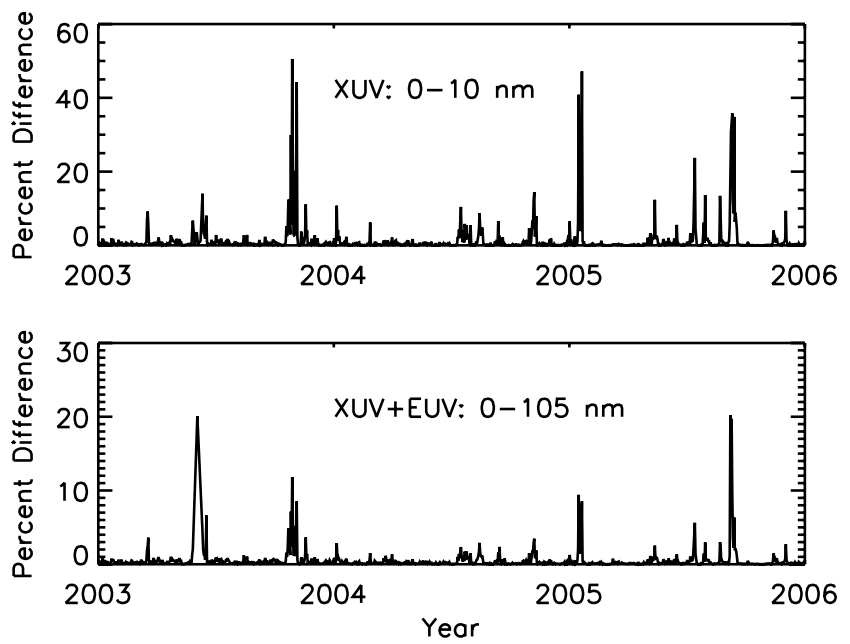

Figure 8. The relative difference in the daily averaged irradiance for two wavelengths bands when the flare contributions are included. This flare contribution shows how much the modeled daily components underestimate the true daily irradiance. 


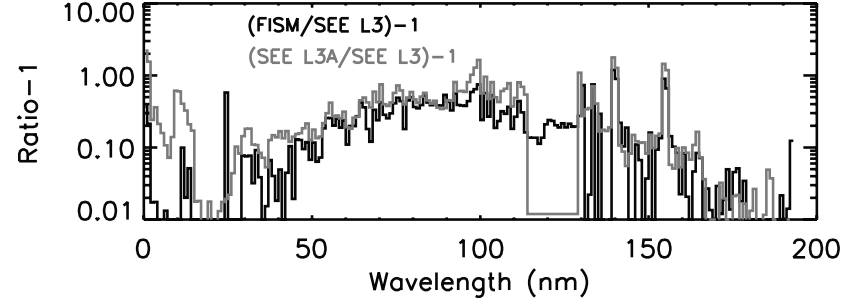

Figure 9. The relative increase estimated by the FISM flare spectrum during the strong impulsive phase flare on 3 November 2003, during the time of the SEE observation. Shown is the ratio-1 of the FISM spectrum (black) and the SEE L3A spectrum (gray) spectra at a time during the flare to the daily averaged SEE L3 data.

especially for the large storm periods such as OctoberNovember 2003 and September 2005, the 0-105 nm daily irradiance is typically underestimated by more than $20 \%$, but is as much as $50 \%$ for the $0-10 \mathrm{~nm}$ wavelengths. The large differences clearly show that there are significant errors introduced by not including the irradiance contributions from flares in modeling the solar XUV and EUV irradiance.

\section{FISM Flare Comparison to Other Data}

[39] There are a limited number of data sets available to compare the FISM flare component results with. The available data sets are from the SEE data, which were used as the basis data set to derive the FISM flare component parameters, as well as the SOHO SEM in the EUV range, and the SORCE SOLSTICE in the FUV range. Future comparisons will become available with the NOAA GOES EUVS, which is currently in orbit but waiting to become operational, as well as the SDO EVE instrument which is scheduled to launch in December 2009.

[40] The first comparison is between FISM and SEE Level 3A (3-min average) data during a flare's impulsive phase. The ratio of the FISM spectrum for a flare on 3 November 2003 (GOES class X3.9) is shown in Figure 9 at the time of the SEE observation that occurred during the impulsive phase. The SEE Level 3A data are the median of the consecutive 10-s integrated spectra that occurred during the observation time. To make the most accurate comparison to the SEE data, the FISM spectrum shown is also the median of all the 10-s spectra that are estimated between the time of the SEE start and stop integration times. The FISM results shown in Figure 9 are the ratio of the flare spectrum that was calculated using the median FISM irradiance spectra during the SEE observations times to the SEE Level 3 daily average spectrum. The SEE observation times were between 0948:45 and 0950:35 UTC for EGS wavelengths greater than $27 \mathrm{~nm}$, and between 0948:03 and 0951:16 UTC for the XPS wavelengths below $27 \mathrm{~nm}$, and this SEE L3A spectrum is also shown as a ratio to the same SEE L3 daily average spectrum for comparison. This figure shows that the FISM spectral response for the impulsive phase of a flare is as expected, with the large increases in the transition region emissions that dominate the middle of the VUV spectrum from 60 to $155 \mathrm{~nm}$. Also shown in Figure 9 is the start of the soft X-ray enhancements as a result of the impulsive phase heating of the flaring loops, showing that the gradual phase has started at the time of the FISM estimation. Detailed comparisons of the FISM spectra to the SEE Level 3A data are not ideal during impulsive phase observations, as both the SEE and the FISM spectra shown here are medians over a time of large and rapid irradiance changes that occur during the impulsive phase and can have large deviations in each individual median spectrum.

[41] A further insight into using the fastest temporal resolution for FISM is that the FISM uncertainty is smallest when using the 3-s GOES 0.1-0.8 nm proxy for comparisons to the SEE observation cadence of $10 \mathrm{~s}$. Figure 10 shows the FISM uncertainty when comparing to the eleven impulsive phase flare observations from SEE for three cases of FISM and GOES temporal resolution. This result clearly shows the improvement in the modeling during the impulsive phase with the faster temporal resolution of both the flare proxy and the FISM output results. The FISM uncertainty is improved in the middle VUV wavelengths by as much as $20 \%$ when using the faster resolution proxy and FISM output.

[42] A second comparison between FISM and SEE Level 3A data is for the gradual phase of the 28 October 2003 GOES X17.2 flare. This flare had a GOES 0.1$0.8 \mathrm{~nm}$ peak around 1110:00 UT, and the EGS observa-

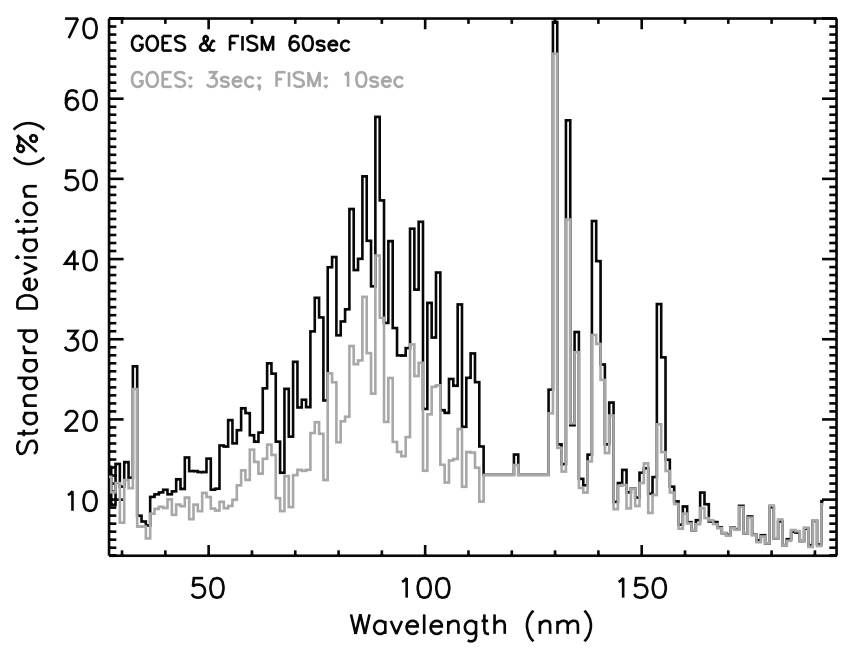

Figure 10. Comparisons of FISM with different temporal resolution to SEE's 11 impulsive phase flare observations. The standard deviation decreases when using the higher temporal resolution of both the GOES $1-8 \AA$ proxy and the FISM output. The black line uses the GOES $1-8 \AA$ 60-s data and FISM 60-s resolution and the gray represents the GOES 3-s resolution with FISM estimations at 10-s intervals. 

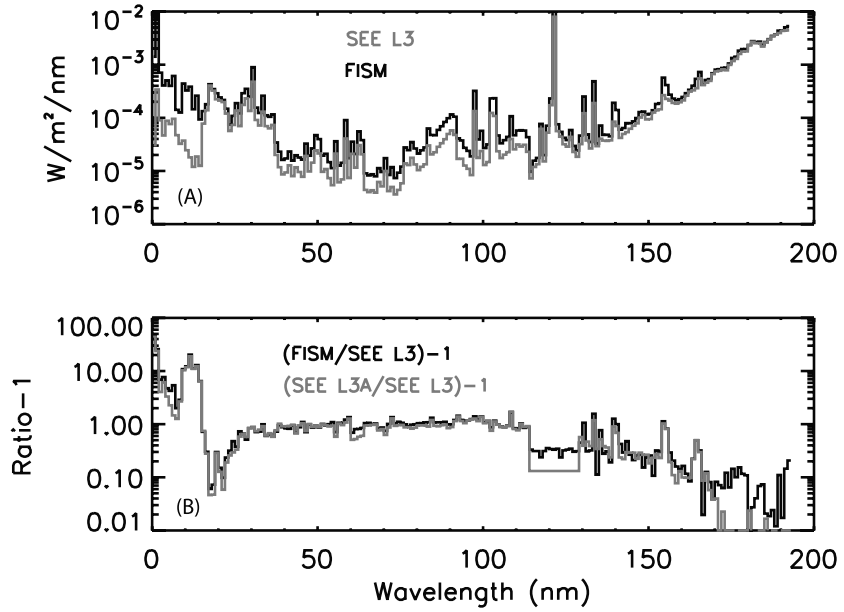

Figure 11. The SEE and FISM flare spectra during the gradual phase of a flare on 28 October 2003 (GOES X17.2). (a) The FISM spectrum (black) and the SEE Level 3 daily median spectrum. (b) The ratio of both the FISM (black) and the SEE L3A (red) flare spectra to the daily averaged SEE L3 data, showing both FISM and SEE have the same amount of irradiance increase over the daily value at all wavelengths for this gradual phase flare.

tion started at 1117:56. The SEE observation occurred more than $7 \mathrm{~min}$ after the peak and thus only the gradual phase should contribute significantly to the FISM flare component results. Unlike the impulsive phase comparisons, more accurate and meaningful comparisons can be made with the SEE Level 3A measurements during the gradual phase as the gradual phase decay rate is more comparable to the 3-min average of the SEE Level 3A data. Figure 11 shows the SEE Level 3 daily median spectrum and also the increased flare irradiance from SEE Level 3A data that are averages over 3-min observations. The FISM spectrum shown is the mean of all the FISM spectra computed between 1117:56 and 1119:35 UT for EGS wavelengths greater than $27 \mathrm{~nm}$ and between 1117:34 and 1120:46 UT for XPS wavelengths less than $27 \mathrm{~nm}$. The FISM irradiance spectrum used in determining the FISM mean spectra are just the 60 -s output resolution for FISM, as that is all that is necessary to accurately model the gradual phase. There is very good agreement between the FISM and SEE data, which is expected as the SEE data for this large flare has a dominant contribution in both the FISM power law fits for the gradual phase flare as well as the FISM flare CLV functions. The FISM spectral profile agrees with the expected results of flare observations during the gradual phase, as there are large responses in the XUV irradiances, typically flat responses across the EUV, and then decreasing responses to the flare for increasing FUV wavelengths. The differences in the longer FUV wavelengths, those greater than $170 \mathrm{~nm}$, are from the solar cycle and solar rotation FISM estimations based on the UARS SOLSTICE data, and the discrepancies show the difference between the SEE and UARS SOLSTICE daily average data.

[43] Figure 12 is a time series of the $154.5 \mathrm{~nm}$ irradiance FISM results for the same flare on 28 October 2003, as well as the SEE Level 3A measurements. There is a very large factor of six increase estimated by FISM for the impulsive phase that is well within expectations derived from SEE observations during the impulsive phase of other smaller flares. The gradual phase of this long duration flare are accurately modeled by FISM, as can be seen by the comparisons of the subsequent SEE Level 3A data points that occurred well after the first SEE data point that was studied in Figure 11.

[44] Results of the 28 October 2003 flare from FISM are also compared to the SEM $26-34 \mathrm{~nm}$ data at $15-\mathrm{s}$ temporal resolution in Figure 13. The SEE 26-34 nm irradiance is approximately $10 \%$ lower then the SEM data [Woods et al., 2005a], and this offset is transferred to the FISM data; therefore the SEM data in Figure 13 are multiplied by a factor of 0.9 to account for this known calibration difference. The absolute values between FISM and SEM results show very good agreement when this discrepancy between the two data sets is considered. The large increase in the SEM data, starting around $1215 \mathrm{UTC}$, is from solar high-energy particles raising the SEM detector background level and does not represent a true irradiance increase. These results show that FISM may be used as a tool to differentiate the actual irradiance increases in the SEM data resulting

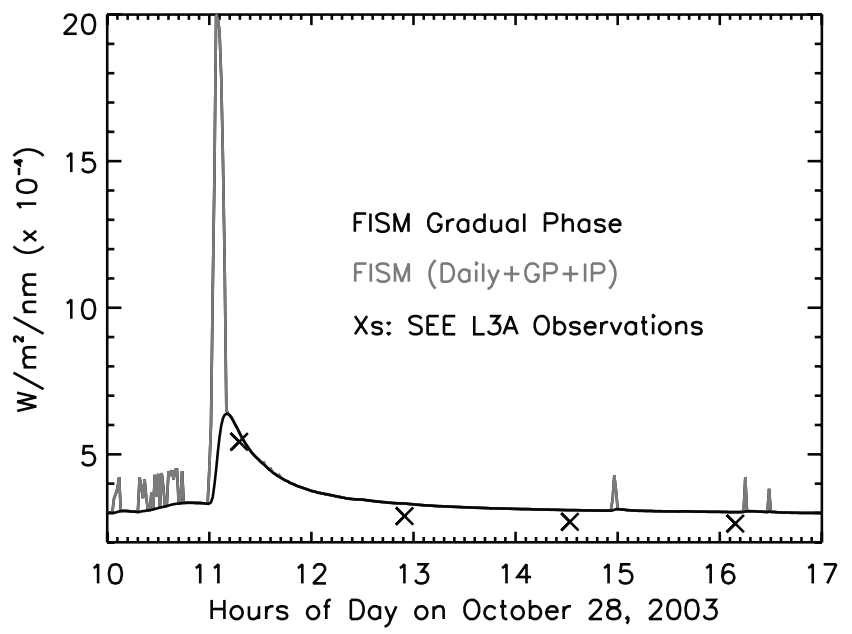

Figure 12. A time series of FISM results and SEE data at $154.5 \mathrm{~nm}$ during the large flare on 28 October 2003 (GOES X17.1). Shown in black is the total FISM flare model that includes the daily average, impulsive phase, and gradual phase components. Also shown are the SEE Level 3A measurements (Xs) and the gradual phase component from FISM (gray). 


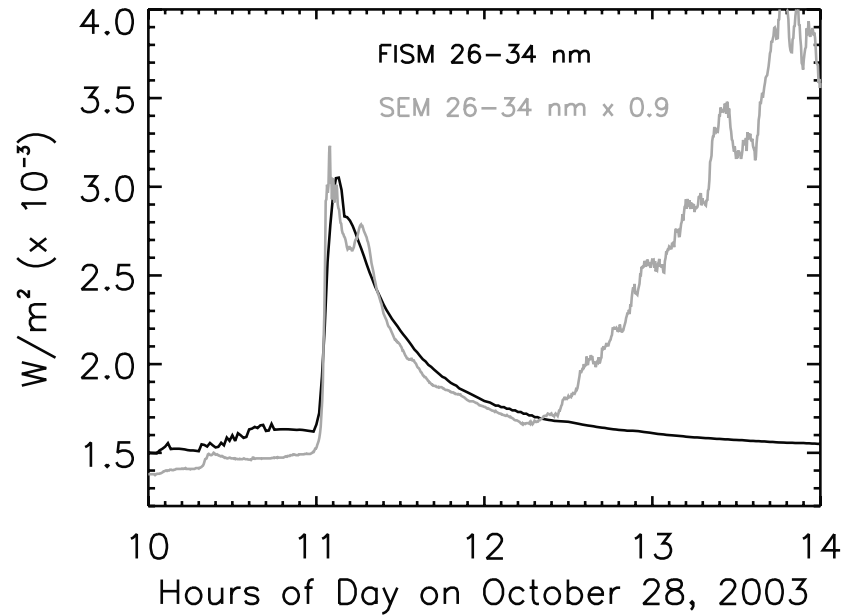

Figure 13. Time series of the 26-34 $\mathrm{nm}$ irradiances from SEM (gray) and FISM (black) for the X17.2 flare that occurred on 28 October 2003. The SEM data is adjusted by a factor of 0.9 to account for the absolute offset of the SEE and SEM data. There is a large increase in the SEM data just after 1200 from solar high-energy particles raising the SEM detector background level.

from flares and those increases that are from highenergy particles.

[45] There is good agreement in the FISM temporal response of the impulsive phase of the flare shown in Figure 13, while the gradual phase peak seems to occur earlier in the FISM results than in SEM as well as being of a lower magnitude. It has been shown by Aschwanden [2004] that the peak irradiance in the gradual phase for cooler emissions occurs at later times. The later peak of the cooler emissions means that the $26-34 \mathrm{~nm}$ band shown here, having formation temperatures ranging from $\log (\mathrm{T})$ of 4.4 to 6.4 , should have the gradual phase emission peak at a later time than the $\log (\mathrm{T})$ of 7.2 for the GOES 0.1-0.8 nm irradiance that is used to model FISM flare irradiances. Unfortunately, for the limited data set provided by SEE and the lower than needed instrument temporal resolution, no conclusions as to the time lag for the VUV wavelengths could be determined from the available data. The delay time of the peaks of the VUV increases during flares is yet another discrepancy that should be resolved when data from SDO EVE become available.

[46] Another comparison of FISM and SEE Level 3A data is shown for the $\mathrm{X} 28+\mathrm{limb}$ flare that occurred on 4 November 2003 as demonstration of the CLV correction used in FISM. The FISM spectra shown in Figure 14 is near the peak of the gradual phase of the flare, but are not as certain as the GOES 0.1-0.8 nm detector saturates at around the X17.2 level. The FISM irradiance for the flare spectra was performed using modeled GOES $0.1-0.8 \mathrm{~nm}$ data during the time of saturation based on the 0-7 $\mathrm{nm}$ channel of the SORCE XPS instrument that observed at a 5-min time cadence throughout the GOES saturation period of the flare. The modeled results are then interpolated onto a $60-\mathrm{s}$ and 3-s temporal grid to replace the saturated GOES values in the respective GOES data set. The expected spectral response profile of the flare during the gradual phase is as expected, and is similar in spectral shape to that of the 28 October 2003 flare discussed previously. In comparison to the 28 October 2003 that occurred in the center of the disk, this flare on 4 November 2003 occurred on the limb and had a much lower response in the EUV and FUV from the center-to-limb darkening of these emissions, while the modeled XUV emissions are still higher. Figures 11 and 14 together demonstrate the ability of FISM to accurately model the CLV corrections for flares that are consistent with the SEE observations.

[47] For the same X28 flare on 4 November 2003, a similar comparison of the FISM estimations to the SOHO SEM measurements is shown in Figure 15, similar to that shown in Figure 13 for the 28 October 2003 flare. This again shows FISM to overall be in very good agreement with the results from the SEM channel. It can also be seen, as stated previously, that the FISM CLV correction also produces accurate estimations for this limb event flare as can be seen by the agreement of the FISM magnitudes in comparison to the SEM data. Another thing that can be seen in this comparison, as
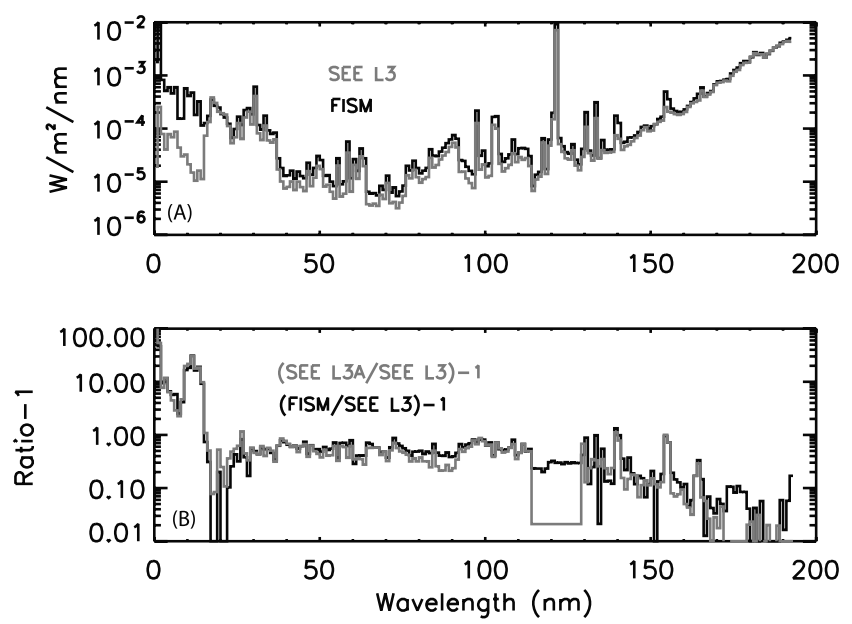

Figure 14. The FISM and SEE spectra during the gradual phase of a flare on 4 November 2003 (GOES X28+). (a) The FISM spectrum (black), and the SEE Level 3 daily average spectrum with flares removed from the average (gray). (b) The ratio of both the FISM (black) and the SEE L3A (gray) flare spectra to the daily averaged SEE L3 data, showing both FISM and SEE have the same amount of irradiance increase over the daily value at all wavelengths for this flare. 


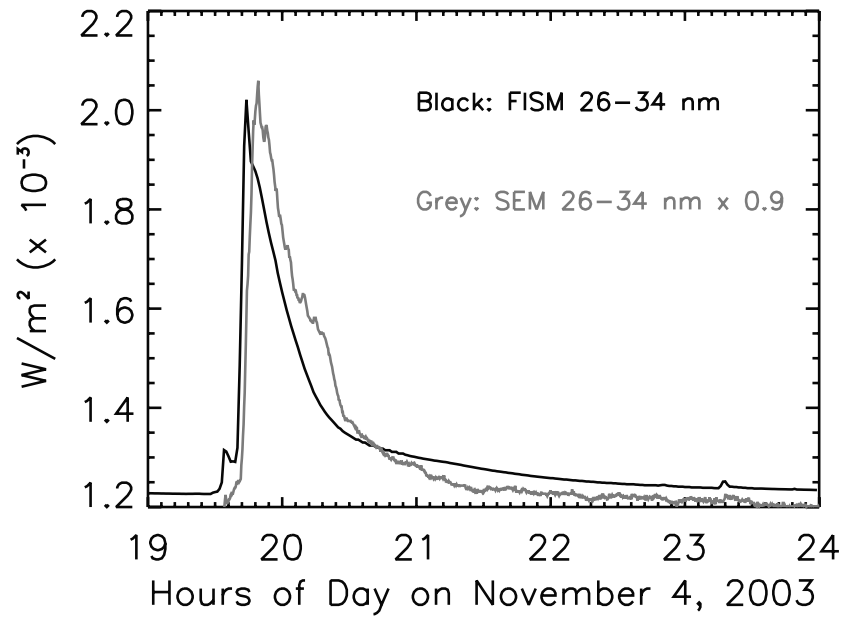

Figure 15. Time series of the $26-34 \mathrm{~nm}$ irradiances from SEM (gray) and FISM (black) for the X28+ flare that occurred on 4 November 2003. The SEM data is adjusted by a factor of 0.9 to account for the absolute offset of the SEE and SEM data.

discussed previously, is the slight offset that can occur in timing of the impulsive phase estimations due to the limitations of only currently having one available impulsive phase proxy, the time derivative of the GOES XRS soft $X$ rays.

[48] A final comparison is between FISM and SORCE SOLSTICE for the bright transition region C IV emission at $154.9 \mathrm{~nm}$. Since SOLSTICE is a spectrometer, it cannot observe the solar spectrum at all FUV wavelengths simultaneously; therefore, SOLSTICE has very few flare observations for comparing to FISM. One large flare that was observed during the impulsive phase by SORCE SOLSTICE is the X10.0 flare that peaked at 2049 UT on 29 October 2003. The higher spectral resolution SOLSTICE measurements are summed from 154.00 to 154.99 to compare to the FISM 1-nm interval centered on $154.5 \mathrm{~nm}$. As SOLSTICE is a spectrometer, measurements within this bandpass are performed at different times, but the duration of the scan across these wavelengths was only $23 \mathrm{~s}$. The results from FISM and SOLSTICE measurements for this flare are shown in Figure 16. The FISM estimate agrees very well with the SOLSTICE preflare observation at 1905 UTC, again showing the accuracy of the FISM daily model component. The fortuitous SOLSTICE observation near the peak of the impulsive phase also shows good agreement, well within the FISM standard deviation, where the percent difference between the two irradiances is $8.83 \%$ and the FISM uncertainty for this wavelength is $34.7 \%$. These comparisons with independent SOLSTICE (and SEM) observations are encouraging that FISM, developed from a few flare observations by SEE, is able

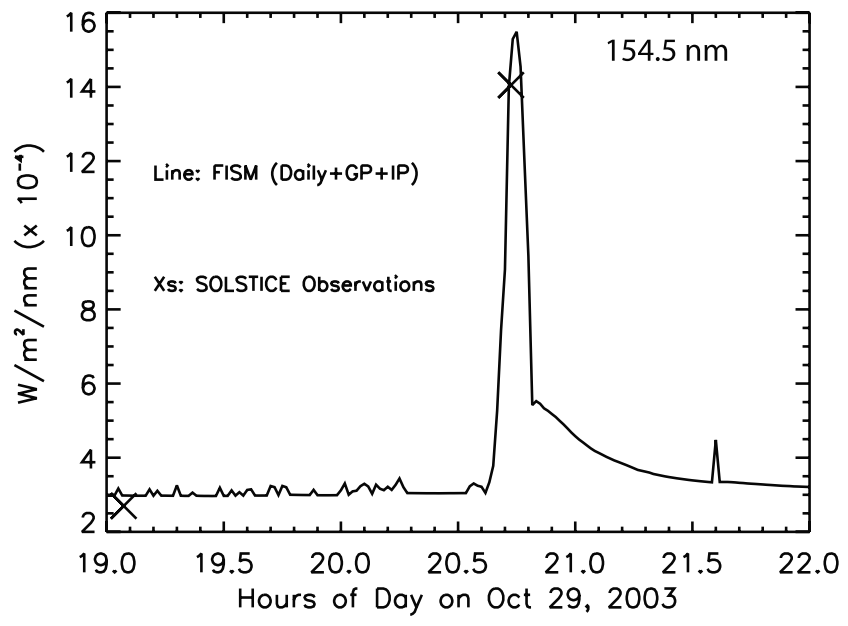

Figure 16. Comparison of the 154-155 nm irradiance from FISM and SORCE SOLSTICE. The percent difference between the FISM and SOLSTICE measurements at the time of the SOLSTICE impulsive phase observation is $8.83 \%$, well within the FISM uncertainty of $34.7 \%$ for this wavelength. The SOLSTICE measurement prior to the onset of the flare (1905) shows good agreement with FISM, once again verifying the FISM daily component is accurately being modeled for this wavelength.

to accurately estimate the solar irradiance variations from flare events.

\section{Discussion}

[49] A comparison of the changes due to the gradual and impulsive phase variations is shown in Figure 17. The solar cycle and solar rotation variations are the same as were covered in the FISM1 paper that discussed the FISM daily components. The flare variations

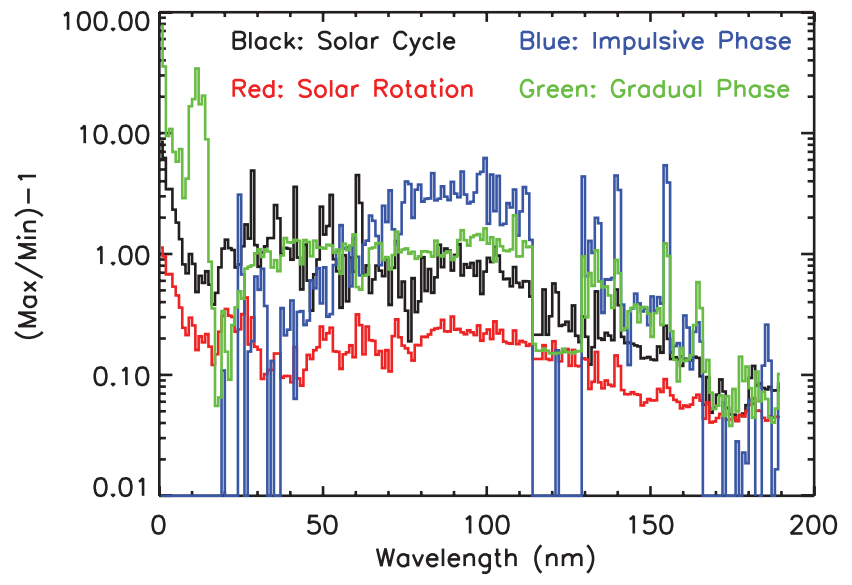

Figure 17. Comparisons of the irradiance variability due to solar cycle (black), solar rotation (red), and the impulsive (blue) and gradual (green) phases of flares. 
use the FISM estimated spectra from the peak of the impulsive and gradual phase variations from the $\mathrm{X} 17$ flare on 28 October 2003, that are divided by the FISM daily component spectrum for that day. This comparison shows that the flare variations are larger than the solar cycle variations for most wavelengths, even though the flare variations last for much shorter timescales from minutes (impulsive phase) to hours (gradual phase). While the solar cycle and solar rotation variations have similar spectral shape, the flare components have uniquely different spectral shapes from each other and from the daily components.

[50] The comparisons of FISM to the SEE, SOLSTICE, and SEM data verify that the solar flare variations are being accurately modeled. The comparisons to the SEM data also show good agreement where the difference falls within the uncertainties of each of the data sets. There are still large uncertainties associated with the FISM flare component in the XUV from the small data set from SEE and SORCE and will hopefully be improved in the future with SDO EVE and GOES EUVS. But, for all wavelengths from $14 \mathrm{~nm}$ up to FISM boundary at $190 \mathrm{~nm}$, FISM is shown to accurately estimate the solar spectrum during solar flares to within $40 \%$. While this uncertainty appears large, the magnitude of flare variations is much larger (factors of $2-100$ ), and FISM is the first flare irradiance model with this level of uncertainty. FISM thus provides the most accurate and complete modeled irradiance data set available of the VUV that includes irradiance variations on all timescales from solar cycles (years) to solar flares (seconds).

\section{Conclusion}

[51] As seen from the results here for flares and in the FISM1 paper for daily variations, FISM is providing accurate results of the solar irradiance variations over timescales ranging from seconds to years. The ability of FISM to accurately model the flare variations may lead to the quantification of the amount of energy deposited into the solar atmosphere during the impulsive phase, as well as the amount of energy that goes into heating the flare loops during the gradual phase. These energy quantizations are not currently known for the VUV wavelengths and are missing when calculating the total energy budget of flares. For example, Woods et al. [2006] show that the total energy of flares is about 10 times more than earlier estimates and that the VUV contribution ranges from $20 \%$ of the total energy for flares near disk center to $100 \%$ for limb flares.

[52] FISM estimations of the solar VUV irradiance can now be used to drive models of the thermosphere, mesosphere, and ionosphere (such as TIE-GCM and WACCM). These models can quantify the magnitude of the neutral and electron density increases in the atmosphere due to solar flares. This can then help answer questions such as "How much will the drag rate of a particular satellite increase for a given size flare?" or "At what size flare will there be a radio communication blackouts?," among may other questions that exist today about how solar flares can affect Earth and the technological systems that people rely on.

[53] FISM data and further information (references, papers, applications, etc.) can be found on the FISM Web site (http://lasp.colorado.edu/lisird/fism.htm) or by contacting the author.

[54] Acknowledgments. This work has been supported by NASA grant NAG5-11408 (TIMED SEE) at the University of Colorado.

\section{References}

Aschwanden, M. J. (2004), Physics of the Solar Corona, Springer, Berlin.

Bailey, S. M., C. A. Barth, M. J. Erickson, R. A. Kohnert, A. W. Merkel, E. M. Rodgers, S. C. Solomon, S. D. Straight, J. E. Vian, and T. N. Woods (1996), Science instrumentations for the Student Nitric Oxide Explorer, Proc. SPIE Int. Soc. Opt. Eng., 2830, 264. Bailey, S. M., T. N. Woods, C. A. Barth, S. C. Solomon, R. Korde, and L. R. Canfield (2000), Measurements of the solar soft X-ray irradiance by the Student Nitric Oxide Explorer: First analysis and underflight calibrations, J. Geophys. Res., 105, 27,179, doi:10.1029/ 2000JA000188.

Baum, W. A., F. S. Johnson, J. J. Oberly, C. C. Rockwood, C. V. Strain, and R. Tousey (1946), Solar ultraviolet spectrum to 88 kilometers, Phys. Rev., 70, 781, doi:10.1103/PhysRev.70.781.

Brueckner, K. L., K. L. Edlow, L. E. Floyd IV, J. L. Lean, and M. E. Vanhoosier (1993), The Solar Ultraviolet Spectral Irradiance Monitor (SUSIM) experiment on board the Upper Atmosphere Research Satellite (UARS), J. Geophys. Res., 98, 10695, doi:10.1029/ 93JD00410.

Chamberlin, P. C., T. N. Woods, and F. G. Eparvier (2007), Flare Irradiance Spectral Model (FISM): Daily component algorithms and results, Space Weather, 5, S07005, doi:10.1029/2007SW000316.

Dennis, B. R., and D. M. Zarro (1993), The Neupert Effect: What can it tell us about the impulsive and gradual phases of solar flares?, Sol. Phys., 146, 177, doi:10.1007/BF00662178.

Garcia, H. A. (1994), Temperature and emission measure from GOES soft X-ray measurements, Sol. Phys., 154, 275, doi:10.1007/ BF00681100.

Hinteregger, H. E., K. Fukui, and B. R. Gilson (1981), Observational, reference, and model data on solar EUV from measurements on AE-E, Geophys. Res. Lett., 8, 1147, doi:10.1029/GL008i011p01147.

Hochedez, J.-F., et al. (2006), LYRA, a solar UV radiometer on Proba2, Adv. Space Res., 2, 202.

Horan, D. M., R. W. Kreplin, and K. P. Dere (1983), Direct measurements of the gradual extreme ultraviolet emission from large solar flares, Sol. Phys., 85, 303, doi:10.1007/BF00148656.

Judge, D. L., et al. (1998), First solar EUV irradiances obtained from SOHO by the CELIAS/SEM, Sol. Phys., 177, 161, doi:10.1023/ A:1004929011427.

Kretzschmar, M., J. Lilensten, and J. Aboudarham (2006), Retrieving the solar EUV spectral irradiance from the observation of 6 lines, Adv. Space Res., 37(2), 341-346.

Lean, J. L., H. P. Warren, J. T. Mariska, and J. Bishop (2003), A new model of solar EUV irradiance variability: 2. Comparisions with empirical models and observations and implications for space weather, J. Geophys. Res., 108(A2), 1059, doi:10.1029/2001JA009238.

McClymont, A. N., and R. C. Canfield (1986), The solar flare extreme ultraviolet to hard X-ray ratio, Astrophys. J., 305, 936, doi:10.1086/ 164307.

Neupert, W. N. (1968), Comparison of solar X-ray line emission with microwave emission during flares, Astrophys. J., 153, L59, doi:10.1086/180220. 
Neupert, W. N. (1989), Transient coronal extreme ultraviolet emission before and during the impulsive phase of a solar flare, Astrophys. J., 344, 504, doi:10.1086/167819.

Priest, E. R. (Ed.) (1981), Solar Flare Magnetohydrodynamics, Gordon and Breach, New York.

Richards, P. G., J. A. Fennelly, and D. G. Torr (1994), EUVAC: A solar EUV flux model for aeronomic calculations, J. Geophys. Res., 99, 8981, doi:10.1029/94JA00518.

Richards, P. G., T. N. Woods, and W. K. Peterson (2006), HEUVAC: A new high resolution solar EUV proxy model, Adv. Space Res., 37(2), 315, doi:10.1016/j.asr.2005.06.031.

Rottman, G. J., T. N. Woods, and T. P. Sparn (1993), Solar-Stellar Irradiance Comparison Experiment I: 1. Instrument design and operation, J. Geophys. Res., 98, 10,667, doi:10.1029/93JD00462.

Thompson, W. T., and P. Brekke (2000), EUV full-Sun imaged spectral atlas using the SOHO coronal diagnostic spectrometer, Sol. Phys., 195, 45, doi:10.1023/A:1005203001242.

Tobiska, W. K. (2004), SOLAR2000 irradiances for climate change research, aeronomy, and space system engineering, Adv. Space Res., 34, 1736, doi:10.1016/j.asr.2003.06.032.

Tobiska, W. K. (2007), Solar2000 v2.30 and SOLARFLARE v1.01: New Capabilities for Space System Operations, Am. Inst. of Aeron. and Astron., Reston, Va.

Tobiska, W. K., T. N. Woods, F. G. Eparvier, R. Viereck, L. Floyd, D. Bower, G. J. Rottman, and O. R. White (2000), The SOLAR2000 empirical solar irradiance model and forcast tool, J. Atmos. Sol. Terr. Phys., 62, 1233, doi:10.1016/S1364-6826(00)00070-5.

Warren, H. P., J. T. Mariska, and J. Lean (1998), A new reference spectrum for the EUV irradiance of the quiet Sun: 1. Emission measure formulation, J. Geophys. Res., 103, 12,077, doi:10.1029/ 98JA00810.

Warren, H. P., J. T. Mariska, and J. Lean (2001), A new model of solar EUV irradiance variability: 1. Model formulation, J. Geophys. Res., 106, 15,745, doi:10.1029/2000JA000282.

Woods, T. N., and G. J. Rottman (2002), Solar ultraviolet variability over time periods of aeronomic interest, in Atmospheres in the Solar System: Comparative Aeronomy, Geophys. Monogr. Ser., vol. 130, edited by M. Mendillo, A. Nagy, and J. H. Waite Jr., p. 221, AGU, Washington, D. C.
Woods, T. N., and G. Rottman (2005), XUV Photometer System (XPS): Solar variations during the SORCE mission, Sol. Phys., 230, 375, doi:10.1007/s11207-005-2555-7.

Woods, T. N., F. Eparvier, S. Bailey, S. C. Solomon, G. Rottman, G. Lawrence, R. Robel, O. R. White, J. Lean, and K. Tobiska (1998a), TIMED solar EUV experiment, Proc. SPIE, 3442, 180, doi:10.1117/12.330255.

Woods, T. N., G. J. Rottman, S. M. Bailey, S. C. Solomon, and J. Worden (1998b), Solar extreme ultraviolet irradiance measurements during solar cycle 22, Sol. Phys., 177, 133, doi:10.1023/ A:1004912310883.

Woods, T. N., F. G. Eparvier, J. Fontenla, J. Harder, G. Kopp, W. E. McClintock, G. Rottman, B. Smiley, and M. Snow (2004), Solar irradiance variability during the October 2003 solar storm period, Geophys. Res. Lett., 31, L10802, doi:10.1029/2004GL019571.

Woods, T. N., F. G. Eparvier, S. M. Bailey, P. C. Chamberlin, J. Lean, G. J. Rottman, S. C. Solomon, W. K. Tobiska, and D. L. Woodraska (2005a), Solar EUV Experiment (SEE): Mission overview and first results, J. Geophys. Res., 110, A01312, doi:10.1029/2004JA010765.

Woods, T. N., G. Rottman, and R. Vest (2005b), XUV Photometer System (XPS): Overview and Calibrations, Sol. Phys., 230, 345, doi:10.1007/s11207-005-4119-2

Woods, T. N., G. Kopp, and P. C. Chamberlin (2006), Contributions of the solar ultraviolet irradiance to the total solar irradiance during large flares, J. Geophys. Res., 111, A10S14, doi:10.1029/2005JA011507.

Woods, T. N., et al. (2008), XUV Photometer System (XPS): Improved solar irradiance algorithm using Chianti spectral models, Sol. Phys., in press.

Worden, J. R., T. N. Woods, and K. W. Bowman (2001), Far-ultraviolet intensities and center-to-limb variations of active regions and quiet Sun using UARS SOLSTICE irradiance measurements and ground-based spectroheliograms, Astrophys. J., 560, 1020, doi:10.1086/323058.

P. C. Chamberlin, F. G. Eparvier, and T. N. Woods, 1234 Innovation Drive, Boulder, CO 80303, USA. (phil.chamberlin@lasp.colorado.edu) 